\title{
Fecal S100A12 as a Biomarker in Behcet's Disease
}

\author{
Cem Şimşek ${ }^{1}$ (D) , Berkan Armağan² (D) , Alper Sarı ${ }^{2}$ (D) , Abdulsamet Erden² (iD , \\ Levent Kılıç (iD , Ali Akdoğan² (D)
}

${ }^{1}$ Hacettepe University Faculty of Medicine, Division of Gastroenterology, Ankara, Turkey ${ }^{2}$ Hacettepe University Faculty of Medicine, Division of Rheumatology, Ankara, Turkey

Cem ŞIMŞSK, M.D.

Berkan ARMAĞAN, M.D.

Alper SARI, M.D.

Abdulsamet ERDEN, M.D.

Levent KILIÇ, M.D.

Ali AKDOĞAN, M.D.

Correspondence: Cem ŞiMŞEK Hacettepe University Faculty of Medicine, Division of Gastroenterology,

Ankara, Turkey

Phone: +905342964684

E-mail: cemgsimsek@gmail.com
ABSTRACT

Background: Gastrointestinal involvement in Behcet's disease impacts morbidity and mortality. The diagnosis of gastrointestinal disease requires comprehensive evaluation with endoscopic and radiologic examinations which is costly and impractical. A biomarker is essential for non-invasive detection. Fecal S100A12 is an established biomarker in gastrointestinal inflammatory diseases and its serum levels are known to increase in Behcet's Disease. In this study, we aimed to tests fecal S100A12 levels in Behcet's patients, its relation with gastrointestinal symptoms to its potential as a biomarker in gastrointestinal involvement.

Methods: We prospectively enrolled 48 cases of Behcet's disease patients fulfilling International Study Group criteria from a university hospital. We excluded patients with other autoimmune conditions, active or recent infection, using NSAIDs and antibiotics. Control group was selected from volunteers who had applied to the rheumatology outpatient clinic. Patients with BD were categorized into 5 groups according to organ involvement. Also we evaluated and recorded disease activity with the BDCAF 2006. Fecal S100A12, fecal calprotectin and acute phase reactants were also collected.

Results: Fecal calprotectin levels were six-fold higher in BD than controls ( $p<0.0001)$. Fecal $\$ 100 \mathrm{~A} 12$ was also two-fold higher albeit without statistical significance $(p=0.132)$. Fecal calprotectin and fecal S100A12 levels were positively correlated $(r: 0.530, p<0.0001)$. Both fecal calprotectin $(138.1 \mu \mathrm{g} / \mathrm{g}$ vs $50.1 \mu \mathrm{g} / \mathrm{g}, \mathrm{p}=0.006)$.) and fecal $\mathrm{S} 100 \mathrm{~A} 12(48.3 \mathrm{ng} / \mathrm{mL}$ vs $19.4 \mathrm{ng} / \mathrm{mL}, \mathrm{p}=0.023)$ were higher in Behçet's patients with gastrointestinal symptoms compared to those without. Fecal calprotectin and fecal S100A12 levels were not correlated with total BDCAF score, CRP and ESR levels.

Conclusion: Fecal S100A12 is correlated with fecal calprotectin and higher in Behcet's patients with gastrointestinal involvement. This is the first study of fecal S100A12 in Behcet's disease and its potential use in Behcet's disease patients with gastrointestinal symptoms.

Keywords: Behcet's disease, gastrointestinal involvement, S100A12, fecal biomarker

\section{Behçet Hastalarında Yeni Biyobelirteç: Fekal S100A12}

ÖZET

Giriş ve amaç : Behçet hastalığında $(\mathrm{BH})$ gastrointestinal tutulum, morbidite ve mortaliteyi etkiler ancak tanısı endoskopik ve radyolojik incelemelerle kapsamlı bir değerlendirme gerektirir. Fekal S100A12, gastrointestinal inflamatuar hastalıklarda kullanılan bir biyobelirteçtir, aynı zamanda serum düzeylerinin Behçet Hastalığında arttığı bilinmektedir, dolayıııyla non-invaziv bir tanı yöntemi olabilir. İşe bu çalışmada, Behçet hastalarında fekal S100A12 düzeylerinin, gastrointestinal semptomlarla ilişkisinin ve dolayısıyla gastrointestinal tutulumda bir biyobelirteç olma potansiyelini araştırmayı amaçladık.

Gereç ve yöntem: Bir üniversite hastanesinden International Study Group kriterlerine uyan 48 Behçet hastasını prospektif olarak değerlendirdik. NSAll'ler ve antibiyotikler kullanan diğer otoimmün durumları, ve enfeksiyonu olan hastaları dışladık. Kontrol grubu Romatoloji polikliniğine başvuran gönüllülerden seçildi. BH olan hastalar organ tutulumlarına göre 5 gruba ayrıldı. Ayrıca BDCAF 2006 ile hastalık aktivitesini değerlendirdik ve kaydettik. Fekal S100A12, fekal kalprotektin ve akut faz reaktanları da çalışldı.

Bulgular: Fekal kalprotektin düzeyleri BH'da kontrollere göre altı kat daha yüksekti( $p<0.0001)$. Fekal $\$ 100 \mathrm{~A} 12$ de istatistiksel olarak BH'da kontrollere göre anlamlı olmasa da iki kat daha yüksekti $(\mathrm{p}=0.132)$. Fekal kalprotektin ve fekal $\mathrm{S} 100 \mathrm{~A} 12$ seviyeleri pozitif korelasyon

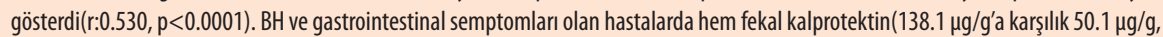
$\mathrm{p}=0.006$ ) hem de fekal $\mathrm{S} 100 \mathrm{~A} 12\left(48.3 \mathrm{ng} / \mathrm{mL} \mathrm{L}^{\prime}\right.$ e karşı $\left.19.4 \mathrm{ng} / \mathrm{mL}, \mathrm{p}=0.023\right)$ olmayanlara göre daha yüksekti. Fekal kal protektin ve fekal S100A12 seviyeleri toplam BDCAF skoru, CRP ve ESR seviyeleri ile korele değildi.

Sonuç: Gastrointestinal semptomları olan Behçet hastalarında fekal S100A12 fekal kalprotektin ile koreledir ve daha yüksektir. Bu, Behçet hastalığında dışıı S100A12'nin ilk çalışmasıdır ve gastrointestinal semptomları olan Behçet hastalı̆ı hastalarında potansiyel kullanımını göstermektedir.

Anahtar Kelimeler: Behçet hastalı̆ıı, gastrointestinal tutulum, S100A12, fekal biyobelirteç 
B ehçet's disease (BD) is a chronic, relapsing multisystem vasculitis that skin, mucosa, joints, eyes, vascular, central nervous system, and the gastrointestinal system (GIS) involvement can be seen in the disease course with time and severity varying patterns of attacks and organ involvements (1). Assessing the activity of BD is challenging since its subclinical organ involvement and subjective nature of symptomatology. Although, acute phase reactants such as C-reactive protein (CRP) and erythrocyte sedimentation rate (ESR) are used in clinical practice to estimate disease activity for various rheumatic inflammatory diseases, those markers perform limited reliability and a reduced utility in $\mathrm{BD}$ patients $(2,3)$. An alternative way is to utilize multimodality scores to monitor disease course. One such tool is Behçet's Disease Current Activity Form (BDCAF) which is a widely established to asses BD overall disease activity $(4,5)$. BDCAF was found to have a good interobserver reliability in British population to assessing general disease activity (5). However, in Turkish populations there was a poor agreement for BDCAF between and within observers for their overall impression of disease activity albeit the score demonstrated limited utility to assess the orogenital ulcers and ocular involvement (6). Therefore, it is an imperative need to identify organ involvements, attacks and overall disease activity in BD.

Gastrointestinal involvement in $\mathrm{BD}(\mathrm{GBD})$ is known to be caused by vasculitic involvement of mesenteric and intramural arterial and venous systems. GBD has different frequencies in Eastern and Western Hemispheres ranging from $1 \%$ to $50 \%$ albeit this data comes from studies with different methodologies. Regardless of its frequency, GBD poses important challenges in the management of $\mathrm{BD}$ as its presence in severe cases, longer time to diagnosis, a challenging differential diagnosis list, and high proportion of complicated cases. Among those, diagnosis of GBD remains a principal issue as current treatments for $B D$ are well known to remission in GBD when diagnosed. Current practice to diagnose GBD relies on upper and lower endoscopic examinations and small bowel imaging when in doubt. This practice relies on the assumption that ileocolonic area is most commonly involved, however it has become clear that isolated small bowel involvement is not infrequent. This poses the question that should every BD patient should be for GBD, and if yes, how?

Use of fecal biomarkers have been a widely utilized way to monitor disease activity and gastrointestinal tract pathologies. The widely established Calprotectin (i.e. S100 8/9) is a dimer of S100A9 and S100A8 and is a calcium binding, pro-inflammatory protein mainly released by activated granulocytes, macrophages and endothelial cells $(7,8)$. S100A12 is another protein from the same family with similar functions and structure, however predominantly expressed by neutrophilic leucocytes (9) which we may pose the question of an additional diagnostic value in BD. These proteins are known to be expressed at sites of inflammation and a higher concentrations are found in various inflammatory sites including serum, feces, synovial fluid, urine and saliva. They are used in the diagnosis, activity and demonstration of gastrointestinal involvement in many rheumatologic or non-rheumatologic diseases, especially inflammatory bowel disease (IBD) (10) (11-13).

In the literature, serum levels of calprotectin and S100A 12 have been studied. Our current knowledge is mostly about disease activation and organ involvement in rheumatologic diseases, while studies about fecal levels of these markers are scarce $(7,13,14)$. In BD patients, serum levels of calprotectin was higher than healthy controls but there was no correlations with the BDCAF (15). Higher fecal calprotectin $(F C)$ level was an independent predictor for intestinal involvement of BD and correlate with the disease activity index of intestinal BD (16). The correlation of the elevated serum levels of S100A12 and BDCAF scores was also shown in BD patients (13). The S100A12 is a reasonable biomarker for $\mathrm{BD}$ as its differential expression by Neutrophils, its demonstrated reliability and feasibility as a fecal marker, and challenges in diagnosing and monitoring gastrointestinal involvement in this population, however has not yet been studied to the best of our knowledge.

The aim of our study was to evaluate both the fecal levels of calprotectin and S100A12 in active and inactive BD patients. We also examined their relationships with gastrointestinal and other symptoms and organ involvements.

\section{METHODS}

\section{Study Population}

Between February 2017 and May 2018, a total of 48 BD patients who were admitted to the Rheumatology Outpatient Clinic of Hacettepe University Hospital were prospectively enrolled in this study. Our study was approved by Hacettepe University Institutional Review Board. All patients with BD fulfilled the International Study Group 
criteria for diagnosis of BD at their time of diagnosis(17). Patients younger than 18 years of age, having other autoimmune conditions, history of malignancy, active or recent infection in past 3 weeks, using non-steroidal antiinflammatory drugs in past 3 weeks and pregnancy were excluded at the time of study enrollment. Control group was selected from 14 healthy volunteers who had applied to the rheumatology outpatient clinic with non-specific pain, without gastrointestinal symptoms and diseases, and without inflammatory diseases sharing the same exclusion criteria. All patients and healthy participants were informed about the study before and consents were obtained before enrollment visit. The study protocol was approved by our institutional review board.

\section{Study design}

Every subject was evaluated by a detailed medical history and a physical examination at the enrollment visit when all participants were thoroughly questioned about signs, symptoms and history of any articular, gastrointestinal, vascular, ocular and neurological involvement. Data regarding demographics, comorbidities, smoking status, disease duration, type of systemic involvement, previous and current medications were obtained from medical records and by face to face interview at that time.

Patients with $\mathrm{BD}$ were categorized into 5 groups according to organ involvement: group 1: mucocutaneous and articular involvement, group 2: ocular involvement, group 3: vascular involvement, group 4: gastrointestinal involvement and group 5: neurologic involvement. BD patients with two or more organ involvement except mucocutaneous and articular involvement were excluded. Remaining 31 patients: 14 patients with mucocutaneous and articular involvement, 12 patients with ocular involvement, and 5 patients with vascular involvement were also separately evaluated as BD with isolated organ involvement.

We evaluated and recorded disease activity with the BDCAF 2006 (5) which was . tested and validated for Turkish patients by Hamuryudan et al (6). Patients were considered to have an active disease if a minimum of two items in 'BDCAF' were observed (15). We also evaluated the BD patients separately for the presence of symptoms indicative of gastrointestinal involvement as follows: oral ulcer, nausea/vomiting/abdominal pain, and diarrhea or frank blood per rectum as gastrointestinal complaint. We also evaluated the $\mathrm{BD}$ patients separately according to BDCAF's domains of nausea/vomiting/abdominal pain, and diarrhea or frank blood per rectum as gastrointestinal complaint.

Conventional inflammatory biomarkers as CRP, ESR, white blood cells, platelet count and haemoglobin were also recorded. CRP and ESR upper normal levels were $0.8 \mathrm{mg} / \mathrm{dL}$ and $20 \mathrm{~mm} / \mathrm{hr}$, respectively.

\section{Measurements of fecal S100A12 and fecal calprotectin concentrations}

Using a disposable stool sampler, approximately $50 \mathrm{mg}$ of fecal specimen from BD patients and controls was collected and immediately frozen at $-80^{\circ} \mathrm{C}$ in a standard refrigerator until further analysis. FC levels were measured by EUROIMMUN Calprotectin ELISA test (Lübeck, Germany) and fecal S100A12 by BioVendor ELISA test (Brno, Czech Republic) according to the manufacturer's instructions. The detection limits of the assays $6.5 \mu \mathrm{g} / \mathrm{g}$ and $0.01 \mathrm{ng} / \mathrm{ml}$, respectively. Cut-off of $50 \mathrm{mg} / \mathrm{kg}$ was used for fecal calprotectin as previously used in similar studies (18).

\section{Statistical analysis}

Statistical analysis was performed using SPSS version 23.0 (SPSS Inc., Chicago, USA). Continuous data were described as mean (standard deviation, SD) or median (IQR) and categorical variables as percentages. Chi-square and Fisher's exact test was used to compare categorical variables. The variables were investigated using visual (histograms, probability plots) and analytical methods to determine whether or not they are normally distributed. Kruskal-Wallis and Mann Whitney $U$ tests were conducted to compare non-normally distributed variables and Bonferroni correction use to adjust for multiple comparisons. One-way ANOVA and Student T test were conducted to compare normally distributed variables and pairwise post-hoc tests were performed using Tukey's test when an overall significance was observed. Correlation of variables was assessed by Spearman correlation coefficient. p value of $<0.05$ was considered as significant. 


\section{RESULTS}

In this study, 48 BD patients and 14 controls were included. There were no statistically significant difference in gender $29(60 \%)$ vs $9(\% 64)$ male as well as the median age of $41.7 \pm 11.1$ vs $39.6 \pm 10.3$ between $B D$ and control groups ( $p=0.794$ and $p=0.532$, respectively). For BD group, median disease duration was 144 (IQR, 12-492) months. Clinical features and disease manifestations of BD patients are summarized in Table 1. Median BDCAF score was 3.0 (IQR, 0-6.0). Regarding the clinical characteristics of $\mathrm{BD}$ oral ulcer was the most common involvement with 48 (100\%) patients, other involvements with decreasing frequency were as follows: papulo-pustular lesion by 36 (75\%), genital ulcer by 30 (63\%), uveitis by $25(52 \%)$, erythema nodosum by 23 (48\%), vascular involvement by 15 (31\%), articular involvement by $14(29 \%)$, neurologic involvement by $8(17 \%)$, and gastrointestinal involvement by $4(8 \%)$.

\section{Table 1. Demographic and clinical characteristics of Behçet} disease patients

\begin{tabular}{|l|c|}
\hline & Behçet disease patients \\
\hline Male, $\mathrm{n}(\%)$ & $29(60)$ \\
\hline Age, mean \pm SD & $41.7 \pm 11.1$ \\
\hline Disease duration (months), median (IQR) & $144(12-492)$ \\
\hline Oral ulcer, $\mathrm{n}(\%)$ & $48(100)$ \\
\hline Genital ulcer, $\mathrm{n}(\%)$ & $30(63)$ \\
\hline Erythema nodosum, $\mathrm{n}(\%)$ & $23(48)$ \\
\hline Papulo-pustular lesion, $\mathrm{n}(\%)$ & $36(75)$ \\
\hline Articular involvement, $\mathrm{n}(\%)$ & $14(29)$ \\
\hline Uveitis, $\mathrm{n}(\%)$ & $25(52)$ \\
\hline Pathergy, $\mathrm{n}$ (\%) & $7 / 15(47)$ \\
\hline Vascular involvement, $\mathrm{n}(\%)$ & $15(31)$ \\
\hline Neurologic involvement, $\mathrm{n}(\%)$ & $8(17)$ \\
\hline Gastrointestinal involvement, $\mathrm{n}(\%)$ & $4(8)$ \\
\hline BDCAF, median (IQR) & $3(0-6)$ \\
\hline BDCAF; Behçet's Disease Current Activity Form & \\
\hline
\end{tabular}

An overview of biomarkers for both BD and control groups were presented in Table 2. Hematologic parameters of hemoglobin concentration, leucocyte and platelet counts were similar in between the groups. ESR were higher in BD compared to controls though both medians were within normal range $(8 \mathrm{~mm} / \mathrm{h}$ vs $2 \mathrm{~mm} / \mathrm{h}, \mathrm{p}=0.028)$, whereas CRP were similar between the groups ( 0.49 vs $0.35 \mathrm{mg} / \mathrm{dL}$ ). Regarding fecal biomarkers, FC levels were approximately six-fold higher in $B D(66.5 \mathrm{vs} 11.6 \mu \mathrm{g} / \mathrm{g}, \mathrm{p}<0.0001)$ than the control group $(p<0.0001)$. Concomitantly, fecal S100A12 was approximately two-fold higher in $\mathrm{BD}$ albeit without statistical significance ( $27.7 \mathrm{vs} 10.2 \mathrm{ng} / \mathrm{mL}, \mathrm{p}=0.132$ )

\begin{tabular}{|l|c|c|c|}
\hline \multicolumn{4}{|l|}{ Table 2. Comparison of demographic data and laboratory } \\
features of patients with BD and controls \\
\hline & $\begin{array}{l}\text { Behçet disease } \\
\text { patients, } \mathrm{n}=48\end{array}$ & $\begin{array}{c}\text { Healthy } \\
\text { controls, } \mathrm{n}=14\end{array}$ & $\mathrm{p}$ \\
\hline $\begin{array}{l}\text { Hemoglobin (g/dL), } \\
\text { mean } \pm \text { SD }\end{array}$ & $13.8 \pm 1.5$ & $14.6 \pm 1.3$ & 0.123 \\
\hline $\begin{array}{l}\text { White blood cell } \\
\text { (mm3), mean } \pm \text { SD }\end{array}$ & $7239 \pm 2002$ & $7592 \pm 2019$ & 0.564 \\
\hline $\begin{array}{l}\text { Platelets (103 mm3), } \\
\text { mean } \pm \text { SD }\end{array}$ & $237 \pm 61$ & $223 \pm 36$ & 0.435 \\
\hline $\begin{array}{l}\text { ESR (mm/h), median } \\
\text { (IQR) }\end{array}$ & $8(3-22)$ & $2(2-10.5)$ & 0.028 \\
\hline $\begin{array}{l}\text { CRP (mg/dl), median } \\
(I Q R)\end{array}$ & $0.49(0.3-1.5)$ & $0.35(0.3-0.4)$ & 0.077 \\
\hline $\begin{array}{l}\text { Fecal Calprotectin } \\
\text { ( } \mu \mathrm{g} / \mathrm{g}), \text { median (IQR) }\end{array}$ & $\begin{array}{c}66.5 \\
(28.8-153.5)\end{array}$ & $11.6(6.1-38.3)$ & $<0.0001$ \\
\hline $\begin{array}{l}\text { Fecal S100A12 (ng/mL), } \\
\text { median (IQR) }\end{array}$ & $27.7(13.7-95.8)$ & $10.2(8.1-30.3)$ & 0.132 \\
\hline ESR; Erythrocyte sedimentation rate, CRP; C-reactive protein & \\
\hline
\end{tabular}

Fecal calprotectin and fecal S100A12 levels were correlated with each other as expected $(r: 0.530, p<0.0001)$. A FC of $50 \mathrm{mcg} / \mathrm{g}$ were considered positive for gastrointestinal inflammation. AUC for fecal S100A 12 to detect FC greater than $50 \mathrm{mcg} / \mathrm{g}$ yielded 0.77 (Cl: $0.65-0.89$ ) AUC. Youden $\mathrm{J}$ Index was 53 for FS to detect FC positivity with specifity $97 \%$ and sensitivity $56 \%$. Neither FC nor fecal S100A12 was correlated with total BDCAF score BD patients with or without gastrointestinal symptoms. They were also not correlated with CRP and ESR levels.

BD patients were also evaluated in terms of overall disease activity as total BDCAF score, as well as presence or absence of gastrointestinal symptoms and oral ulcers as included in BDCAF. When we compare our patients disease activity according to $\mathrm{BDCAF} \geq 2$, there were $37(77 \%)$ active and 11 (23\%) inactive patients and fecal markers and serum AFR were shown in the Table 3. There was no statistical significance in fecal and serum activity markers between active or inactive BD groups. When we compared our patients according to gastrointestinal complaint + OA, there were 35 (73\%) positive and 13 (27\%) negative patients and fecal, and serum disease activity markers were shown in the Table 4. Although BD patients with 
gastrointestinal complaint $+\mathrm{OA}$ had higher levels of fecal and serum activity markers, there was no statistically significant between BD patients with and without gastrointestinal symptoms + OA. When we compared our patients according to only gastrointestinal symptoms, there were $30(63 \%)$ positive and 18 (37\%) negative patients. Fecal, and serum disease activity markers were shown in the Table 5. BD patients with gastrointestinal symptoms had a higher FC levels (138.1 $\mu \mathrm{g} / \mathrm{g}$ (IQR:52-296) vs 50.1 $\mu \mathrm{g} / \mathrm{g}$ (IQR:22-100); $\mathrm{p}=0.006)$. Similarly, fecal S100A12 levels in $\mathrm{BD}$ patients with gastrointestinal symptoms were also higher than without those (48.3 ng/mL (IQR: 21-164) vs $19.4 \mathrm{ng} / \mathrm{mL}$ (IQR:12-54); $\mathrm{p}=0.023$ ) with statistical significance in pairwise comparison. There was no significant differences in serum disease activity marker levels.

Table 3. Fecal calprotectin, fecal S100A12 and CRP levels in active (according to $\mathrm{BDCAF} \geq 2$ ) and inactive Behçet disease patients and healthy control

\begin{tabular}{|l|c|c|c|c|c|} 
& $\begin{array}{c}\text { Active } \\
\mathrm{BD}, \mathrm{n}=37\end{array}$ & $\begin{array}{c}\text { Inactive } \\
\mathrm{BD}, \mathrm{n}=11\end{array}$ & $\begin{array}{c}\text { Healthy } \\
\text { control }\end{array}$ & $\mathrm{p}$ & \multirow{2}{*}{$\mathrm{p}^{*}$} \\
\hline $\begin{array}{l}\text { Hemoglobin }(\mathrm{g} / \\
\mathrm{dL}) \text {, mean } \pm \mathrm{SD}\end{array}$ & $13.9 \pm 1.4$ & $\begin{array}{c}13.5 \pm \\
2.0\end{array}$ & $\begin{array}{c}14.6 \pm \\
1.3\end{array}$ & 0.198 & \\
\cline { 1 - 4 } White blood cell & $7256 \pm$ & $7181 \pm$ & $7592 \pm$ & \multirow{2}{*}{0.843} &
\end{tabular}

\begin{tabular}{|c|c|c|c|c|c|}
\hline$(\mathrm{mm} 3)$, mean $\pm \mathrm{SD}$ & 2209 & 1125 & 2019 & 0.843 & \\
\hline $\begin{array}{l}\text { Platelets ( } 103 \\
\mathrm{~mm} 3), \text { mean } \pm \text { SD }\end{array}$ & $239 \pm 58$ & $231 \pm 73$ & $223 \pm 36$ & 0.682 & \\
\hline $\begin{array}{l}\text { Fecal Calprotectin } \\
(\mu \mathrm{g} / \mathrm{g}), \text { median } \\
(\mathrm{IQR})\end{array}$ & $\begin{array}{c}65.2 \\
(31-156)\end{array}$ & $\begin{array}{c}93 \\
(26-151)\end{array}$ & $\begin{array}{c}11.6 \\
(6.1-38.3)\end{array}$ & 0.001 & 0.532 \\
\hline $\begin{array}{l}\text { Fecal S100A12 } \\
(\mathrm{ng} / \mathrm{mL}), \text { median } \\
(\mathrm{IQR})\end{array}$ & $\begin{array}{c}25.2 \\
(13-126)\end{array}$ & $\begin{array}{c}32 \\
(11-57)\end{array}$ & $\begin{array}{c}10.2 \\
(8-32)\end{array}$ & 0.254 & \\
\hline $\begin{array}{l}\mathrm{CRP}(\mathrm{mg} / \mathrm{dl}), \\
\text { median (IQR) }\end{array}$ & $\begin{array}{c}0.5 \\
(0.3-1.5)\end{array}$ & $\begin{array}{c}0.69 \\
(0.2-1.9)\end{array}$ & $\begin{array}{c}0.35 \\
(0.3-0.4)\end{array}$ & 0.208 & \\
\hline $\begin{array}{l}\text { ESR (mm/h), } \\
\text { median (IQR) }\end{array}$ & $\begin{array}{c}7 \\
(3.5-13.5)\end{array}$ & $\begin{array}{c}22.5 \\
(3-47)\end{array}$ & $\begin{array}{c}2 \\
(2-10.5)\end{array}$ & 0.065 & \\
\hline
\end{tabular}

* $p$ values: Comparison of the active and inactive Behçet disease patients with eachother (*p values for multiple comparisons with Bonferroni correction $<0.017)$

$\mathrm{BD}$; Behçet disease, CRP; C-reactive protein, ESR; Erythrocyte sedimentation rate
Table 4. Fecal calprotectin, fecal S100A12 and CRP levels in Behçet disease patients with and without gastrointestinal symptoms + oral ulcer and healthy control

\begin{tabular}{|c|c|c|c|c|c|}
\hline & $\begin{array}{c}\text { BD with } \\
\text { GIS+OA, } \\
n=35\end{array}$ & $\begin{array}{c}\mathrm{BD} \\
\text { without } \\
\text { GIS+OA, } \\
\mathrm{n}=13\end{array}$ & $\begin{array}{l}\text { Healthy } \\
\text { control }\end{array}$ & $p$ & $p^{*}$ \\
\hline $\begin{array}{l}\text { Hemoglobin (g/ } \\
\mathrm{dL} \text { ), mean } \pm \text { SD }\end{array}$ & $\begin{array}{c}13.6 \pm \\
1.5\end{array}$ & $\begin{array}{c}14.4 \pm \\
1.5\end{array}$ & $\begin{array}{c}14.6 \pm \\
1.3\end{array}$ & 0.097 & \\
\hline $\begin{array}{l}\text { White blood cell } \\
(\mathrm{mm} 3) \text {, mean } \\
\pm \mathrm{SD}\end{array}$ & $\begin{array}{c}7131 \pm \\
2014\end{array}$ & $\begin{array}{c}7530 \pm \\
2021\end{array}$ & $\begin{array}{c}7592 \pm \\
2019\end{array}$ & 0.705 & \\
\hline $\begin{array}{l}\text { Platelets (103 } \\
\text { mm3), mean } \\
\pm \mathrm{SD}\end{array}$ & $233 \pm 56$ & $247 \pm 75$ & $223 \pm 36$ & 0.563 & \\
\hline $\begin{array}{l}\text { Fecal } \\
\text { Calprotectin } \\
(\mu g / g), \text { median } \\
(\text { IQR) }\end{array}$ & $\begin{array}{c}68.8 \\
(43-159)\end{array}$ & $\begin{array}{c}40.1 \\
(12-114)\end{array}$ & $\begin{array}{c}11.6 \\
(6-38)\end{array}$ & $<0.0001$ & 0.051 \\
\hline $\begin{array}{l}\text { Fecal S100A12 } \\
(\mathrm{ng} / \mathrm{mL}), \text { median } \\
\text { (IQR) }\end{array}$ & $\begin{array}{c}30.6 \\
(17-140)\end{array}$ & $\begin{array}{c}16.0 \\
(5-51)\end{array}$ & $\begin{array}{c}10.2 \\
(8-30)\end{array}$ & 0.049 & 0.059 \\
\hline $\begin{array}{l}\mathrm{CRP}(\mathrm{mg} / \mathrm{dl}), \\
\text { median (IQR) }\end{array}$ & $\begin{array}{c}0.61 \\
(0.3-1.5)\end{array}$ & $\begin{array}{c}0.45 \\
(0.3-0.8)\end{array}$ & $\begin{array}{c}0.35 \\
(0.3-0.4)\end{array}$ & 0.143 & \\
\hline $\begin{array}{l}\text { ESR (mm/h), } \\
\text { median (IQR) }\end{array}$ & $\begin{array}{c}11 \\
(4-23)\end{array}$ & $5(2-13)$ & $2(2-10)$ & 0.017 & 0.068 \\
\hline
\end{tabular}

$\mathrm{p}^{*}$ values : Comparison of the Behçet disease patients with and without gastrointestinal symptoms + oral ulcer. (* $p$ values for multiple comparisons with Bonferroni correction $<0.017$ )

BD; Behçet disease, GIS; Gastrointestinal symptoms, CRP; C-reactive protein,

ESR; Erythrocyte sedimentation rate

One patient had 3 and 16 patients had 2 different organ involvement in our study. So, we had 48 BD patients with 66 organ involvement (including mucocutaneous and articular involvement). When we excluded the BD patients with multiple organ involvement, 14 patients with mucocutaneous and articular involvement, 12 patients with ocular involvement, and 5 patients with vascular involvement were separately evaluated as BD with isolated organ involvement. Differences in fecal and serum markers of disease activity parameters and total score of BDCAF according to $\mathrm{BD}$ organ involvement were presented in Table 6. There was no statistical significant differences among these 3 BD groups with isolated organ involvement. Comparison of $\mathrm{BD}$ patients with isolated organ involvements according to gastrointestinal symptoms + OA, and only gastrointestinal symptoms of BDCAF domains were shown in the table 8 and 9, respectively. There were no statistical significant differences in these 3 BD groups with isolated organ involvement in terms of GIS+OA symptoms, and only GIS symptoms or not. 
Table 5. Fecal calprotectin, fecal S100A12 and CRP levels in Behçet disease patients with and without gastrointestinal symptoms (without oral ulcer) and healthy control

\begin{tabular}{|c|c|c|c|c|c|}
\hline & $\begin{array}{l}\text { BD with } \\
\text { GIS, } \\
n=30\end{array}$ & $\begin{array}{l}\text { BD } \\
\text { without } \\
\text { GIS, } \\
n=18\end{array}$ & $\begin{array}{l}\text { Healthy } \\
\text { control }\end{array}$ & $p$ & $\mathrm{p}^{*}$ \\
\hline $\begin{array}{l}\text { Hemoglobin (g/ } \\
\mathrm{dL} \text { ), mean } \pm \mathrm{SD}\end{array}$ & $\begin{array}{c}13.6 \pm \\
1.7\end{array}$ & $\begin{array}{c}14.0 \pm \\
1.4\end{array}$ & $\begin{array}{c}14.6 \pm \\
1.3\end{array}$ & 0.235 & \\
\hline $\begin{array}{l}\text { White blood cell } \\
(\mathrm{mm} 3) \text {, mean } \\
\pm \mathrm{SD}\end{array}$ & $\begin{array}{c}6883 \pm \\
2260\end{array}$ & $\begin{array}{c}7453 \pm \\
1837\end{array}$ & $\begin{array}{c}7592 \pm \\
2019\end{array}$ & 0.541 & \\
\hline $\begin{array}{l}\text { Platelets }(103 \\
\mathrm{mm} 3) \text {, mean } \\
\pm \mathrm{SD}\end{array}$ & $\begin{array}{c}232 \pm \\
62\end{array}$ & $\begin{array}{c}240 \pm \\
61\end{array}$ & $\begin{array}{c}223 \pm \\
36\end{array}$ & 0.665 & \\
\hline $\begin{array}{l}\text { Fecal } \\
\text { Calprotectin } \\
(\mu g / g), \text { median } \\
(\text { (IQR) }\end{array}$ & $\begin{array}{c}138.1 \\
(52-296)\end{array}$ & $\begin{array}{c}50.1 \\
(22-100)\end{array}$ & $\begin{array}{c}10.9 \\
(5-42)\end{array}$ & $<0.0001$ & 0.006 \\
\hline $\begin{array}{l}\text { Fecal S100A12 } \\
(\mathrm{ng} / \mathrm{mL}), \text { median } \\
(\mathrm{IQR})\end{array}$ & $\begin{array}{c}48.3 \\
(21-164)\end{array}$ & $\begin{array}{c}19.4 \\
(12-54)\end{array}$ & $\begin{array}{c}10.0 \\
(7-29)\end{array}$ & 0.024 & 0.023 \\
\hline $\begin{array}{l}\mathrm{CRP}(\mathrm{mg} / \mathrm{dl}) \text {, } \\
\text { median (IQR) }\end{array}$ & $\begin{array}{c}0.41 \\
(0.3-0.8)\end{array}$ & $\begin{array}{c}0.55 \\
(0.3-1.6)\end{array}$ & $\begin{array}{c}0.32 \\
(0.3-0.4)\end{array}$ & 0.116 & 0.288 \\
\hline $\begin{array}{l}\text { ESR (mm/h), } \\
\text { median (IQR) }\end{array}$ & $9(4-21)$ & $8(3-22)$ & $5(2-11)$ & 0.149 & 0.693 \\
\hline \multicolumn{6}{|c|}{$\begin{array}{l}\mathrm{p}^{*} \text { values: Comparison of the Behçet disease patients with and without } \\
\text { gastrointestinal symptoms. }{ }^{*} \mathrm{p} \text { values for multiple comparisons with } \\
\text { Bonferroni correction }<0.017) \\
\text { BD; Behçet disease, GIS; Gastrointestinal symptoms, CRP; C-reactive protein, } \\
\text { ESR; Erythrocyte sedimentation rate }\end{array}$} \\
\hline
\end{tabular}

\begin{tabular}{|c|c|c|c|c|}
\hline & $\begin{array}{l}\text { Mucocutaneous } \\
\text { and articular } \\
(n=14)\end{array}$ & $\begin{array}{l}\text { Ocular } \\
(\mathrm{n}=12)\end{array}$ & $\begin{array}{c}\text { Vascular } \\
(\mathrm{n}=5)\end{array}$ & $p$ \\
\hline $\begin{array}{l}\text { Fecal Calprotectin } \\
(\mu \mathrm{g} / \mathrm{g}), \text { median } \\
(\mathrm{IQR})\end{array}$ & $79.4(27-122)$ & $\begin{array}{c}60.0 \\
(23-150)\end{array}$ & $\begin{array}{c}70.9 \\
(37-190)\end{array}$ & 0.322 \\
\hline $\begin{array}{l}\text { Fecal S100A12 } \\
(\mathrm{ng} / \mathrm{mL}), \text { median } \\
(\mathrm{IQR})\end{array}$ & $16.7(9-56)$ & $\begin{array}{c}28.9 \\
(15-151)\end{array}$ & $\begin{array}{c}49.3 \\
(18-122)\end{array}$ & 0.320 \\
\hline CRP, median (IQR) & $0.56(0.3-1.9)$ & $\begin{array}{c}0.38 \\
(0.3-1.5)\end{array}$ & $\begin{array}{c}0.83 \\
(0.4-1.5)\end{array}$ & 0.462 \\
\hline ESR, median (IQR) & $5(2-15)$ & $11.5(3-22)$ & $13(6-28)$ & 0.729 \\
\hline $\begin{array}{l}\text { BDCAF, median } \\
\text { (IQR) }\end{array}$ & $2.5(1.8-3.5)$ & $3(2-4)$ & $3(2-5)$ & 0.448 \\
\hline \multicolumn{5}{|c|}{$\begin{array}{l}\text { CRP; C-reactive protein, ESR; Erythrocyte sedimentation rate, BDCAF; Behçet's } \\
\text { Disease Current Activity Form }\end{array}$} \\
\hline
\end{tabular}

Thirty-seven (77\%) patients used colchicine and 21 (44\%) patients used one of the immunosuppressive therapies. Although there was no statistically significant in both, patients undergoing colchicine therapy had a lower level of FC and fecal S100A12 than without colchicine therapy, in contrast, patients undergoing immunosuppressive therapies were higher levels of FC and fecal S100A12 than without immunosuppressive therapies.

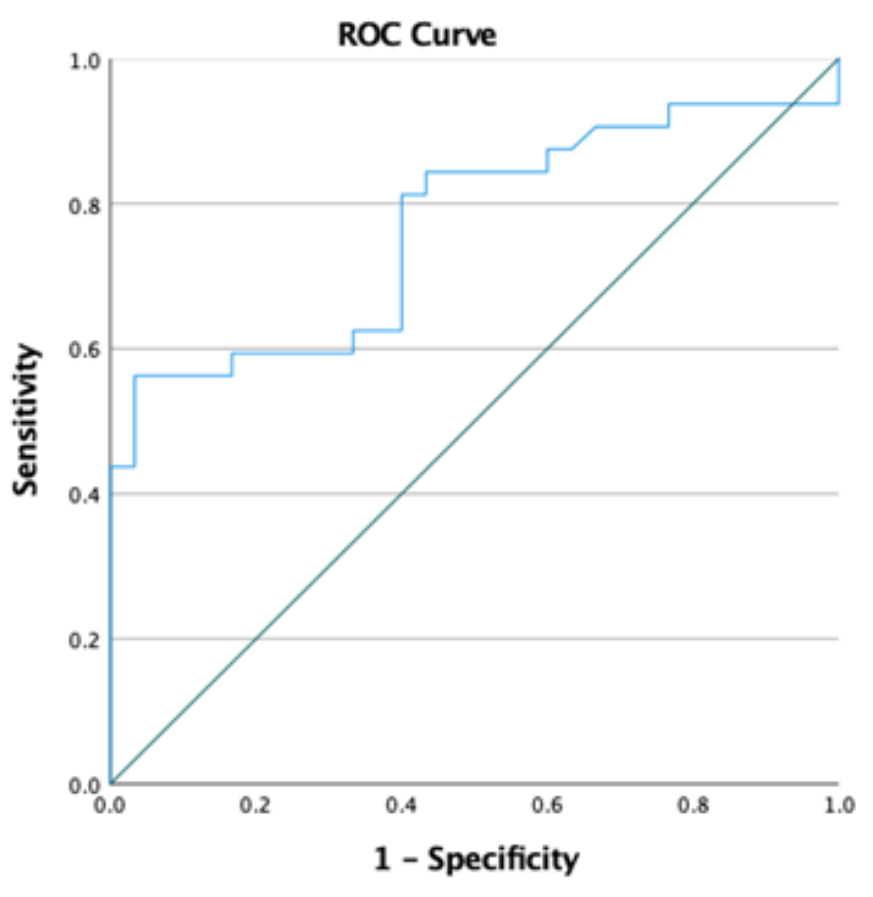

Diagonal segments are produced by ties.

Figure 1. Area under rule operator curve for fecal S100A12 levels in Behçet disease to detect FC greater than $50 \mathrm{mcg} / \mathrm{g}$ 
Table 7. Fecal calprotectin, fecal S100A12 levels in active and inactive Behçet disease groups (GIS+OA)

\begin{tabular}{|c|c|c|c|c|c|c|}
\hline & \multicolumn{3}{|c|}{ Fecal S100A12 (ng/mL) } & \multicolumn{3}{|c|}{ Fecal Calprotectin $(\mu \mathrm{g} / \mathrm{g})$} \\
\hline & $\begin{array}{l}\text { BD with GIS } \\
\text { symptom+OA }\end{array}$ & $\begin{array}{l}\text { BD without GIS } \\
\text { symptom+OA }\end{array}$ & $p$ & $\begin{array}{l}\text { BD with GIS } \\
\text { symptom+OA }\end{array}$ & $\begin{array}{l}\text { BD without GIS } \\
\text { symptom+OA }\end{array}$ & $p$ \\
\hline $\begin{array}{l}\text { Mucocutaneous and } \\
\text { articular }(n=14)\end{array}$ & $18.3(9-97)$ & $15.5(6-23)$ & 0.524 & $79.4(47-122)$ & $56.0(8-283)$ & 0.620 \\
\hline Ocular $(n=12)$ & $27.7(19-37)$ & $11.5(6.6-12.7)$ & 0.116 & $49.2(34-62))$ & $8.8(5-36)$ & 0.229 \\
\hline $\operatorname{Vascular}(n=5)^{*}$ & $95.8(75-420)$ & $24.7(5-44)$ & & $182.5(143-319)$ & $16.2(4-28)$ & - \\
\hline
\end{tabular}

Table 8. Fecal calprotectin, fecal S100A12 levels in active and inactive Behçet disease groups (GIS)

\begin{tabular}{|l|c|c|c|c|c|c|}
\hline & \multicolumn{3}{|c|}{ Fecal S100A12 $(\mathrm{ng} / \mathrm{mL})$} & \multicolumn{3}{c|}{ Fecal Calprotectin ( $\mu \mathrm{g} / \mathrm{g})$} \\
\cline { 2 - 7 } & BD with GIS symptom & BD without GIS symptom & $\mathrm{p}$ & BD with GIS symptom & BD without GIS symptom & $\mathrm{p}$ \\
\hline $\begin{array}{l}\text { Mucocutaneous and } \\
\text { articular }(\mathrm{n}=14)\end{array}$ & $34.2(9-158)$ & $15.5(8-36)$ & 0.571 & $92.5(57-286)$ & $79.2(20-114)$ & 0.396 \\
\hline Ocular $(\mathrm{n}=12)$ & $27.7(15-1319)$ & $19.4(11-31)$ & 0.518 & $49.2(34-273)$ & $43.8(19-62)$ & 0.644 \\
\hline Vascular $(\mathrm{n}=5) *$ & $420(96-746)$ & $44.3(25-49)$ & & $319(182-456)$ & $28.3(16-66)$ & - \\
\hline *Statistical comparison was not applied due to the small number of patients.
\end{tabular}

Table 9. Fecal calprotectin, fecal S100A12 levels in Behçet disease using or not colchicine and immunosuppresive drugs

\begin{tabular}{|c|c|c|c|c|c|c|}
\hline & $\begin{array}{c}\text { BD with } \\
\text { colchicine, } n=37\end{array}$ & $\begin{array}{l}\text { BD without } \\
\text { colchicine, } n=11\end{array}$ & $\mathrm{p}$ & $\begin{array}{c}\text { BD with } \\
\begin{array}{c}\text { immunosuppressive, } \\
n=21\end{array}\end{array}$ & $\begin{array}{c}\text { BD withuot } \\
\text { immunosuppressive, } \\
n=27\end{array}$ & $\mathrm{p}$ \\
\hline Fecal Calprotectin $(\mu \mathrm{g} / \mathrm{g})$, median (IQR) & $65.2(26-131)$ & $97.9(28-283)$ & 0.411 & $68.4(41-213)$ & $58.0(21-98)$ & 0.149 \\
\hline Fecal S100A12 (ng/mL), median (IQR) & $25.7(15-62)$ & $30.6(7-141)$ & 0.922 & $43.7(13-155)$ & $19.4(14-45)$ & 0.299 \\
\hline
\end{tabular}

\section{DISCUSSION}

In this study, FC levels were six-fold higher in BD than the control group ( $p<0.0001)$ and fecal S100A12 was two-fold higher albeit without statistical significance $(p=0.132)$. In terms of classical AFR, ESR were higher in BD compared to controls though both medians were within normal range 0.028), whereas CRP were similar between the groups. Although there was no difference when active BD patients were determined according to total score, as expected, both fecal calprotectin and fecal S100A12 were higher in Behçet's patients with gastrointestinal symptoms compared to those without. Fecal calprotectin and fecal S100A12 levels were correlated with each other but not with total BDCAF score, CRP and ESR levels. On the other hand, FC and fecal S100A12 levels were similar between BD patients using or not colchicine and immunosuppressive drugs.

$\mathrm{BD}$ is a multisystem vasculitis with involvement or virtually any vascular field thus organ system as well as hemostatic abnormalities(19). BD has varying prevalence over different geographical regions, and in areas with a high prevalence such as Turkey, disease constitutes an important cause of morbidity. Current research efforts in $\mathrm{BD}$ are concentrated to: Firstly to elucidate pathogenesis of the disease, secondly to develop clinical tools and imaging protocols for diagnosing different organ involvements in their earlier courses, and lastly to implement newer agents to BD management. Despite the vast improvement in all these areas, there is a long way ahead for $\mathrm{BD}$ and their providers.

Gastrointestinal involvement in BD has varying frequency in different geographical regions spanning from $1 \%$ in Western to $50 \%$ in Eastern Hemisphere, albeit this difference can be owed to different methodologies and disease characteristics in these populations. Gastrointestinal involvement in BD can be in any region in the oropharyngeal area and gastrointestinal tract, oral ulcers and ileocecal areas are well-known to be characteristic as well as nearly pathognomonic for GBD. 
Nevertheless, recent improvements in gastrointestinal, especially small bowel imaging and fecal biomarkers studies pointed out that there might be considerable ongoing inflammation in these parts of the tractus. This new perspective deserves attention as a false negative test for gastrointestinal involvement can cause a failure to induce remission and increase the risk of a serious complication such as perforation or bleeding.

Several studies evaluated the diagnosis of GBD with newer imaging methods and fecal biomarkers. Two studies revealed that up $90 \%$ of symptomatic BD patients with negative upper plus lower endoscopies can have ulcers in their small bowels $(20,21)$. This underlines the importance of multimodality approach for the detection of gastrointestinal involvements presumably with serologic and fecal biomarkers. Anti-Saccharomyces cerevisiae antibodies (ASCA), IgM alpha-enolase antibody, and Interleukin-12 $B$ was suggested as potential serologic biomarkers for $\mathrm{GBD}$, however none has gained wide-spread acceptance (22-24).

S100A 12 shows promise as a candidate biomarker in GBD for several reasons. Firstly, neutrophilic inflammation is a hallmark of vasculitic lesions in gastrointestinal $B D$ and also the main source of detectable fecal S100A12 (25). Secondly, like calprotectin, S100A12 is detectable with ELISA after 7 days in fecal material thus stands out as a feasible diagnostic method. Thirdly, S100A12 demonstrated successful diagnostic performances in other gastrointestinal pathologies and becoming closer to be implemented into clinical practice (26).

Our BD cohort are well established and GBD subgroup is comparable to the previous knowledge with $8 \%$ frequency. In this study, we did not use GBD as a separate cohort, but utilized a cross-sectional study design with prospective involvement study design to understand the overall diagnostic performance of S100A 12 in disease activity and $\mathrm{Gl}$ involvement. About $8 \%$ of our patients had $\mathrm{Gl}$ symptoms. As a limitation, we did not perform endoscopic and imaging gastrointestinal evaluation in our patients. This is because this study is designed as a pilot for further studies and it is well known that none of the current methods have enough sensitivity to exclude GBD alone especially in asymptomatic patients. S100A12 deserves further effort to be compared to current diagnostic utilities. In this prospective enrollment, 15 of 48 (29\%) BD patients demonstrated $\mathrm{FC}$ levels greater than $53 \mathrm{ng} / \mathrm{mL}$. In 48 patients, 18 had abdominal symptoms. FC levels were three-fold higher in symptomatic BD group when compared to asymptomatic BDs. FC levels did not any correlation with BDCAF, or serum acute phase biomarkers, as expectable from a fecal biomarker.

In conclusion, although we could not found any correlation between total BDCAF and fecal markers in our study, they have seemed better than classical AFR in showing BD activity and this difference was more pronounced in those with gastrointestinal symptoms. To the best of our knowledge, this is the first study to evaluate fecal S100A 12 in BD. Fecal S100A12 demonstrated promising distribution among BD patients and presumably will be of dramatic use to diagnose and follow-up gastrointestinal involvement in BD. Also this is the first study showing S100A 12 in addition to fecal calprotectin in Behcet's disease patients with gastrointestinal symptoms.

\section{ACKNOWLEDGEMENTS}

This study was supported by Hacettepe University Scientific Research Fund (THD-2017-13174).

\section{References}

1. Hatemi G, Yazici Y, Yazici H. Behcet's syndrome. Rheum Dis Clin North Am. 2013;39(2):245-61.

2. Sota J, Vitale A, Rigante $D$, Orlando I, Lucherini OM, Simpatico A, et al. Correlation of Serum Amyloid-A Levels, Clinical Manifestations, Treatment, and Disease Activity in Patients with Behcet's Disease. Isr Med Assoc J. 2018;20(8):517-21.

3. Melikoglu M, Topkarci Z. Is there a relation between clinical disease activity and acute phase response in Behcet's disease? Int J Dermatol. 2014;53(2):250-4.

4. Lawton G, Bhakta BB, Chamberlain MA, Tennant A. The Behcet's disease activity index. Rheumatology (Oxford). 2004;43(1):73-8.

5. Bhakta BB, Brennan $P$, James TE, Chamberlain MA, Noble BA, Silman AJ. Behcet's disease: evaluation of a new instrument to measure clinical activity. Rheumatology (Oxford). 1999;38(8):728-33.

6. Hamuryudan V, Fresko I, Direskeneli H, Tenant MJ, Yurdakul S, Akoglu T, et al. Evaluation of the Turkish translation of a disease activity form for Behcet's syndrome. Rheumatology (Oxford). 1999;38(8):734-6.

7. Kopec-Medrek M, Widuchowska M, Kucharz EJ. Calprotectin in rheumatic diseases: a review. Reumatologia. 2016;54(6):306-9.

8. Manolakis AC, Kapsoritakis AN, Tiaka EK, Potamianos SP. Calprotectin, calgranulin C, and other members of the s100 protein family in inflammatory bowel disease. Dig Dis Sci. 2011;56(6):1601-11.

9. Heida A, Van de Vijver E, van Ravenzwaaij D, Van Biervliet S, Hummel TZ, Yuksel Z, et al. Predicting inflammatory bowel disease in children with abdominal pain and diarrhoea: calgranulin-C versus calprotectin stool tests. Arch Dis Child. 2018;103(6):565-71.

10. Johne B, Fagerhol MK, Lyberg T, Prydz H, Brandtzaeg $P$, Naess-Andresen $\mathrm{CF}$, et al. Functional and clinical aspects of the myelomonocyte protein calprotectin. Mol Pathol. 1997;50(3):113-23.

11. Foell D, Roth J. Proinflammatory S100 proteins in arthritis and autoimmune disease. Arthritis Rheum. 2004;50(12):3762-71. 
12. Pietzsch J, Hoppmann S. Human S100A12: a novel key player in inflammation? Amino Acids. 2009;36(3):381-9.

13. Han EC, Cho SB, Ahn KJ, Oh SH, Kim J, Kim DS, et al. Expression of Pro-inflammatory Protein S100A12 (EN-RAGE) in Behcet's Disease and Its Association with Disease Activity: A Pilot Study. Ann Dermatol. 2011;23(3):313-20.

14. Skef W, Hamilton MJ, Arayssi T. Gastrointestinal Behcet's disease: a review. World J Gastroenterol. 2015;21(13):3801-12.

15. Oktayoglu P, Mete N, Caglayan M, Bozkurt M, Bozan T, Em S, et al. Elevated serum levels of calprotectin (MRP8/MRP14) in patients with Behcet's disease and its association with disease activity and quality of life. Scand J Clin Lab Invest. 2015;75(2):106-12.

16. Kim DH, Park Y, Kim B, Kim SW, Park SJ, Hong SP, et al. Fecal calprotectin as a non-invasive biomarker for intestinal involvement of Behcet's disease. J Gastroenterol Hepatol. 2017;32(3):595-601.

17. Criteria for diagnosis of Behcet's disease. International Study Group for Behcet's Disease. Lancet. 1990;335(8697):1078-80.

18. Fauny $M, D$ 'amico F, Bonovas $S$, Netter $P$, Danese $S$, Loeuille $D$, et al. Faecal calprotectin for the diagnosis of bowel inflammation in patients with rheumatological diseases: a systematic review. Journal of Crohn's and Colitis. 2020;14(5):688-93.

19. Koşar A, Öztürk M, Haznedaroğlu I, Karaaslan Y. Hemostatic parameters in Behçet's disease: a reappraisal. Rheumatology international. 2002;22(1):9-15.

20. Hamdulay S, Cheent K, Ghosh C, Stocks J, Ghosh S, Haskard D. Wireless capsule endoscopy in the investigation of intestinal Behçet's syndrome. Rheumatology. 2008;47(8):1231-4.

21. Neves FS, Fylyk SN, Lage LV, Ishioka S, Goldenstein-Schainberg C, Sakai $\mathrm{P}$, et al. Behçet's disease: clinical value of the video capsule endoscopy for small intestine examination. Rheumatology international. 2009;29(5):601-3.

22. Choi CH, Kim TI, Kim BC, Shin SJ, Lee SK, Kim WH, et al. AntiSaccharomyces cerevisiae antibody in intestinal Behçet's disease patients: relation to clinical course. Diseases of the colon \& rectum. 2006;49(12):1849-59.

23. Shin SJ, Kim BC, Kim TI, Lee SK, Lee KH, Kim WH. Anti-alpha-enolase antibody as a serologic marker and its correlation with disease severity in intestinal Behçet's disease. Digestive diseases and sciences. 2011;56(3):812-8.

24. Lee HW, Chung SH, Moon CM, Che X, Kim SW, Park SJ, et al. The correlation of serum IL-12B expression with disease activity in patients with inflammatory bowel disease. Medicine. 2016;95(23).

25. Hayasaki N, Ito M, Suzuki T, Ina K, Ando T, Kusugami K, et al. Neutrophilic phlebitis is characteristic of intestinal Behçet's disease and simple ulcer syndrome. Histopathology. 2004;45(4):377-83.

26. Carvalho A, Lu J, Francis JD, Moore RE, Haley KP, Doster RS, et al. S100A12 in Digestive Diseases and Health: A Scoping Review. Gastroenterology Research and Practice. 2020;2020. 